Jeffrey R. Capadona ${ }^{1,2 \star}$, Andrew J. Shoffstall ${ }^{1,2}$ and Joseph J. Pancrazio ${ }^{3}$

${ }^{1}$ Department of Biomedical Engineering,

Case Western Reserve University, School of

Engineering, Cleveland, OH, USA. ${ }^{2}$ Advanced

Platform Technology Center, L. Stokes Cleveland

VA Medical Center, Rehabilitation Research and

Development, Cleveland, OH, USA. ${ }^{3}$ Department of

Bioengineering, The University of Texas at Dallas,
Richardson, TX, USA.

${ }^{*}$-mail:jrc35@case.edu

Published online: 25 February 2019

https://doi.org/10.1038/s41563-019-0312-9

References

1. Ajiboye, A. B. et al. Lancet 389, 1821-1830 (2017).

2. Barrese, J. C. et al. J. Neural Eng. 10, 066014 (2013).

3. Goss-Varley, M. et al. Sci. Rep. 7, 15254 (2017).

4. Jorfi, M., Skousen, J. L., Weder, C. \& Capadona, J. R. J. Neural Eng. 12, $011001(2015)$

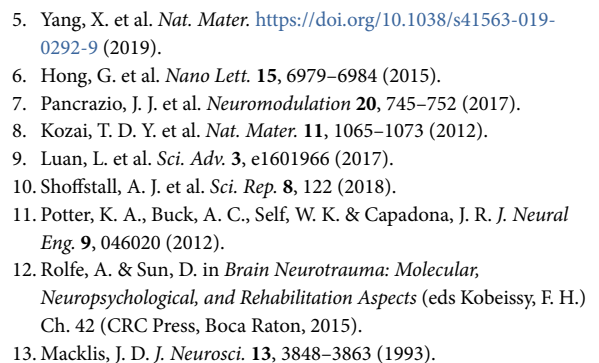

\title{
SURFACE SCIENCE
}

\section{Fast movement in a crowd}

Transport mechanisms of adsorbate molecules across surfaces can influence catalyst reactivity. Now, Joost Wintterlin and colleagues report the results of a study of oxygen adatom transport across the $\mathrm{Ru}(0001)$ surface, but importantly at the high adsorbate coverage that can occur in industrial processes (Science 363, 715-718; 2019).

Catalytic reactions on a surface happen via two mechanisms: either one molecule adsorbed on the surface reacts with another molecule in the gas phase, or both molecules are adsorbed that then diffuse across the surface to encounter each other. The latter typically occurs for $\mathrm{CO}$ oxidation on Pt group metals, a commonly used catalytic model. Surface transport is assumed to be fast and so not rate-limiting, and indeed at low coverages the activation energy for diffusion is low because encounters with other surface species, which can increase diffusion activation energies, are rare. However, at high coverages it was not known whether this assumption of fast transport is correct.

The team performed high-speed scanning tunnelling microscopy imaging, at 10-50 frames per second, of diffusion trajectories of $\mathrm{O}$ adatoms on the $\mathrm{Ru}(0001)$ surface fully covered with CO molecules (left panel, imaging at 12 frames per second). Ruthenium is the least active catalyst of the Pt group and so diffusion can be studied without the interference of $\mathrm{CO}$ oxidation. The $\mathrm{O}$ adatom (red sphere) binds to one of three hexagonal close-packed (hcp) sites around a surface $\mathrm{Ru}$ atom (grey spheres) that does not bind a CO molecule (blue panel). Most of the time the $\mathrm{O}$ adatom hops around the three hcp sites (left panel, inset, green arrows).

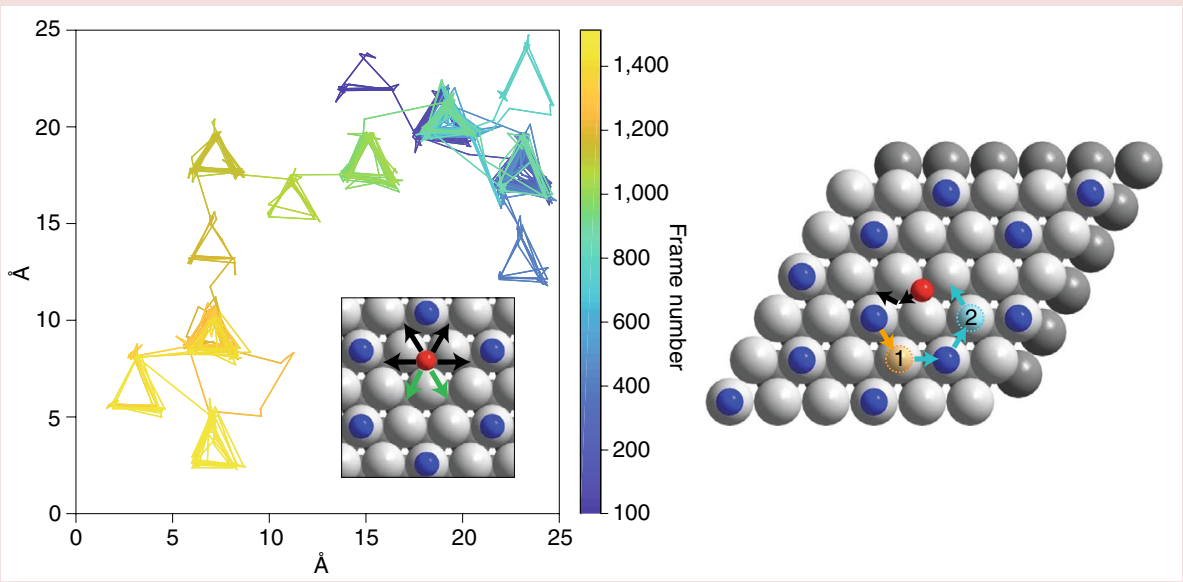

Credit: AAAS

Occasionally, however, $\mathrm{O}$ adatom jumps from a triangular cage to a neighbouring triangular cage were observed (left panel, inset, black arrows); this involved CO exchange in the other direction. A statistical model was developed, allowing extraction of activation energies and demonstrating that only these two processes were needed to describe $\mathrm{O}$ adatom transport. However, transport of the $\mathrm{O}$ adatom is more complicated than naively assumed; it is neither a random walk nor a simple vacancy mechanism.

To shed light on this, the team performed density functional theory calculations of possible transport mechanisms. By far the best fit with experimental activation energies was found for a model where the $\mathrm{CO}$ molecule moves first (right panel, orange arrow). As the team explain, this 'opens a door' that then enables the $\mathrm{O}$ adatom to transport to the neighbouring hcp site with a lower activation energy (right panel, black arrows). Oxygen transport relies on a local density fluctuation of the $\mathrm{CO}$ adsorbates; although the surface is fully covered the density is not high. What is particularly striking is that the frequency of $\mathrm{O}$ adatom exchange is fast, only 3.5 times slower than for an empty surface.

It would be interesting to see if this mechanism also applies for other adsorbates, other metals of the Pt group, and whether this also occurs for higher densities. As it is, though, this work demonstrates that surface adsorbate transport on highly occupied surfaces can be surprisingly rapid.

Stephen Shevlin

Published online: 18 April 2019 https://doi.org/10.1038/s41563-019-0353-0 